\title{
Metabolic and Hormonal Changes After Laparoscopic Roux-en-Y Gastric Bypass and Sleeve Gastrectomy: a Randomized, Prospective Trial
}

\author{
Ralph Peterli • Robert E. Steinert • \\ Bettina Woelnerhanssen - Thomas Peters • \\ Caroline Christoffel-Courtin • Markus Gass • \\ Beatrice Kern • Markus von Fluee • Christoph Beglinger
}

Published online: 22 February 2012

(C) The Author(s) 2012. This article is published with open access at Springerlink.com

\begin{abstract}
Background The mechanisms of amelioration of glycemic control early after laparoscopic Roux-en-Y gastric bypass (LRYGB) or laparoscopic sleeve gastrectomy (LSG) are not fully understood.

Methods In this prospective, randomized 1-year trial, outcomes of LRYGB and LSG patients were compared, focusing on possibly responsible mechanisms. Twelve patients were randomized to LRYGB and 11 to LSG. These non-diabetic patients were investigated before and 1 week, 3 months, and 12 months after surgery. A standard test meal was given after an overnight fast, and blood samples were collected before, during, and after food intake for hormone profiles (cholecystokinin
\end{abstract}

Clinical Trial Registration: www.ClinicalTrials.gov. NCT00356213

R. Peterli $(\bowtie) \cdot$ M. Gass $\cdot$ B. Kern $\cdot$ M. von Fluee

Department of Surgery, St. Claraspital,

4016 Basel, Switzerland

e-mail: Ralph.Peterli@Claraspital.ch

R. E. Steinert

Clinical Research Center, Department of Biomedicine,

University Hospital,

4031 Basel, Switzerland

B. Woelnerhanssen

Department of Surgery, University Hospital,

4031 Basel, Switzerland

T. Peters $\cdot$ C. Christoffel-Courtin

Department of Medicine, St. Claraspital,

4016 Basel, Switzerland

C. Beglinger

Department of Gastroenterology, University Hospital,

4031 Basel, Switzerland
(CCK), ghrelin, glucagon-like peptide 1 (GLP-1), peptide YY (PYY)).

Results In both groups, body weight and BMI decreased markedly and comparably leading to an identical improvement of abnormal glycemic control (HOMA index). Postsurgery, patients had markedly increased postprandial plasma GLP-1 and PYY levels $(p<0.05)$ with ensuing improvement in glucose homeostasis. At 12 months, LRYGB ghrelin levels approached preoperative values. The postprandial, physiologic fluctuation returned, however, while LSG ghrelin levels were still markedly attenuated. One year postoperatively, CCK concentrations after test meals increased less in the LRYGB group than they did in the LSG group, with the latter showing significantly higher maximal CCK concentrations ( $p<0.012$ vs. LRYGB).

Conclusions Bypassing the foregut is not the only mechanism responsible for improved glucose homeostasis. The balance between foregut (ghrelin, CCK) and hindgut (GLP-1, PYY) hormones is a key to understanding the underlying mechanisms.

Keywords Metabolic surgery - Glycemic control · Laparoscopic Roux-en-Y gastric bypass · Laparoscopic sleeve gastrectomy · Gut hormones · Cholecystokinin (CCK) - Ghrelin · Glucagon-like peptide 1 (GLP-1) · Peptide YY (PYY)

\section{Introduction}

Bariatric surgery is the most successful weight loss therapy for morbid obesity, achieving markedly improved glycemic control (including diabetes resolution in most patients) and 
satiety hormone balance [1-3]. Roux-en-Y gastric bypass (RYGB) is effective for treating morbid obesity, inducing a marked and sustained weight loss, even extending to $>10$ years [1, 4-8]. Many surgeons therefore consider RYGB as the bariatric procedure of choice, and nowadays, a laparoscopic approach is most often undertaken (LRYGB) [1, 9-11].

Laparoscopic sleeve gastrectomy (LSG) is a newer approach, initially applied to superobese patients with severe co-morbidities where initial weight loss is intended to enable a later, definitive LRYGB or biliopancreatic diversion duodenal switch as part of a staged concept [12]. Recently published prospective studies compared results from a sole LSG operation to LRYGB [13-17]. Equal early weight loss, markedly improved glucose homeostasis, and increased insulin, glucagon-like peptide 1 (GLP-1), and peptide YY (PYY) levels after both procedures were reported, though LSG seemed easier to perform and associated with fewer complications $[15,17]$. The mechanisms of weight loss following bariatric surgery include restriction, malabsorption, and humoral changes [3, 17-22]. Bypass procedures induce hormonal changes that ameliorate or even lead to complete remission of type 2 diabetes mellitus [3, 18-21]. In this present 1-year prospective, randomized trial, we compared LRYGB and LSG outcomes and investigated potential mechanisms responsible for the observed weight loss and metabolic effects, focusing on the main two hypotheses: foregut vs. hindgut theory $[19,20]$.

\section{Materials and Methods}

\section{Patients}

All studies were performed according to the principles of the Declaration of Helsinki. The Local Research and Ethics Committee in Basel approved the study. The included patients are a subgroup of an ongoing "Swiss Multicenter Bypass or Sleeve Study" (SM-BOSS, NCT00356213), comparing LSG and LRYGB with regard to primary endpoints, effectiveness and safety, to be closed as soon as 100 patients per group have been operated. In this present special group analysis, only non-diabetic patients from our center were consecutively included for logistic reasons. Table 1 provides baseline demographics of the present study. All patients were informed of the risks and benefits of each procedure and provided written, informed consent. Computer-generated random numbers in a sealed envelope determined the type of surgery (LRYGB or LSG). All operations were performed laparoscopically and by the same surgeon. The LRYGB technique included a small gastric pouch with a $25-\mathrm{mm}$ circular pouch-jejunostomy to a $150-\mathrm{cm}$ antecolic Roux limb and an exclusion of $50 \mathrm{~cm}$ of biliopancreatic limb. The LSG was
Table 1 Baseline demographics

\begin{tabular}{lccc}
\hline Parameter & $\begin{array}{l}\text { LRYGB } \\
(n=12)\end{array}$ & $\begin{array}{l}\text { LSG } \\
(n=11)\end{array}$ & $p$ value \\
\hline Male/female & $3 / 9$ & $3 / 8$ & 0.90 \\
Age (years) & $41.4 \pm 10.1$ & $35.2 \pm 10.7$ & 0.18 \\
Fasting insulin $(\mu \mathrm{U} / \mathrm{mL})$ & $30.0 \pm 16.9$ & $28.2 \pm 16.5$ & 0.79 \\
Fasting glucose $(\mathrm{mmol} / \mathrm{L})$ & $5.8 \pm 0.7$ & $5.7 \pm 1.0$ & 0.67 \\
HOMA index & $8.0 \pm 5.1$ & $7.5 \pm 5.5$ & 0.84 \\
HbA1c $(\%)$ & $5.7 \pm 0.3$ & $5.6 \pm 0.6$ & 0.72 \\
Weight $(\mathrm{kg})$ & $133.3 \pm 30.8$ & $120.2 \pm 19.8$ & 0.23 \\
BMI $\left(\mathrm{kg} / \mathrm{m}^{2}\right)$ & $47.6 \pm 6.8$ & $44.7 \pm 5.3$ & 0.25 \\
\hline
\end{tabular}

Data are presented as means \pm SD. No significant differences between the two groups were detected

done along a 35-F bougie from the angle of His to approximately $3-4 \mathrm{~cm}$ orally to the pylorus.

\section{Study Design}

In this randomized, prospective, parallel group trial, all patients underwent complete evaluation before the respective bariatric operation and during follow-up, including medications, nutritional behavior, anthropometric and clinical parameters, and blood sampling for glucose, triglycerides, cholesterol, and other laboratory tests.

For meal studies, subjects were admitted to the Clinical Research Center before the operation and 1 week and 3 and 12 months after the operation. After fasting overnight (at least $10 \mathrm{~h}$ ), an antecubital vein catheter was inserted for phlebotomy. After taking the fasting sample, a 424-kcal $(1,775 \mathrm{~kJ})$ liquid test meal containing $15 \mathrm{~g}$ carbohydrates, $25 \mathrm{~g}$ proteins, and $28 \mathrm{~g}$ fat was served to stimulate hormone release. Blood was drawn at the following times: $-15,0$ (corresponding to commencing meal intake), 15, 30, 45, 60, 120 , and $180 \mathrm{~min}$. Samples (10 ml/withdrawal) were collected into EDTA tubes containing aprotinin at a final concentration of $500 \mathrm{KIU} / \mathrm{mL}$ of blood and a DPP-IV inhibitor; samples were immediately processed and kept on ice to retard peptide breakdown. After centrifugation at $4{ }^{\circ} \mathrm{C}$, plasma samples were kept frozen at $-20^{\circ} \mathrm{C}$ until analysis.

\section{Hormones}

The following hormones were investigated: cholecystokinin (CCK), GLP-1, PYY, insulin, and ghrelin. CCK concentrations were measured using a sensitive radioimmunoassay based on an antiserum that recognizes the sulfated tyrosine residue of all CCK molecules, but has little cross-reactivity with sulfated gastrin $(<1 \%)$ and no cross-reaction with unrelated gastrointestinal peptides. Plasma samples were 
extracted with ethanol, and ${ }^{125}$ I-CCK-8 was used as a label. The lowest concentration currently measurable was $0.6 \mathrm{pmol} / \mathrm{L}$ plasma, using CCK-8 as a standard (details previously described [23]).

The lowest ghrelin level detectable by the commercially available kit (Linco Research Inc. St. Charles, 63304 MO, USA) used is $93 \mathrm{pg} / \mathrm{mL} / 100 \mu \mathrm{L}$ sample. At $1 \mathrm{ng} / \mathrm{mL}$, the intra- and inter-assay coefficients of variation were $10.0 \%$ and $14.7 \%$, respectively [15].

GLP-1 was measured with a commercially available ELISA kit (Linco Research Inc.); the assay used is highly specific for measuring active GLP-1 but does not detect other GLP-1 forms (e.g., 1-36 amide, 1-37, 9-36 amide, or 9-37). This assay sequentially (1) captures active GLP-1 with a monoclonal antibody (binds specifically to the $\mathrm{N}$-terminal region of active GLP-1 molecules); (2) removes unbound materials; (3) binds an anti-GLP-1-alkaline phosphatase detection conjugate to immobilized GLP-1; (4) removes unbound conjugate; and (5) quantifies bound detection conjugate by adding methyl umbelliferyl phosphate which, with alkaline phosphatase, forms fluorescent umbelliferone. Since the degree of fluorescence is directly proportional to the active GLP-1 concentration, the latter is interpolated from a curve using reference standards of known active GLP-1 concentrations. The intraand inter-assay variabilities were below $9 \%$ and $13 \%$, respectively. The lowest level of GLP-1 currently detectable is 0.25 $\mathrm{pmol} / \mathrm{L}$ (100 $\mu \mathrm{L}$ plasma sample) [24].

PYY was measured with a commercially available kit (Linco Research Inc.). The guinea pig antibody displays $100 \%$ cross-reactivity with human PYY1-36 and human PYY3-36, but not with human pancreatic polypeptide, NPY, leptin, or ghrelin). $\left[{ }^{125} \mathrm{I}\right] \mathrm{PYY}$, purified by HPLC (specific activity $302 \mu \mathrm{Ci} / \mu \mathrm{g}$ ), was used as a label. The lowest level of PYY currently detectable is $10 \mathrm{pg} / \mathrm{mL}(100 \mu \mathrm{L}$ plasma sample). The intra- and inter-assay variabilities were below $9 \%$ and $9 \%$, respectively [15].

The lowest level of insulin currently detectable with the commercial radioimmunoassay (Cisbio International, 30200 Bagnols, France) used is $4.6 \mu \mathrm{U} / \mathrm{mL}(100 \mu \mathrm{L}$ sample). The intra- and inter-assay coefficients of variation were $12.2 \%$ and $9.0 \%$, respectively [15]. Blood glucose concentrations were measured using a commercial hexokinase-glucose-6-phosphate-dihydrogenase method (Roche, 4070 Basel, Switzerland).

\section{Statistical Analysis}

Data analysis was performed using the statistical software package, SPSS for Windows V. 15.0 (SPSS Inc., Chicago, IL 60606-6306, USA). Values are reported as means \pm SEM. Descriptive statistics were used for demographic variables, such as age, weight, height, and BMI. Hormones were analyzed by calculating time courses, the area under the plasma concentration time profiles (AUC), and maximum plasma concentrations (Cmax). The general linear model procedure of repeated measures ANOVA using simple contrast was used to test for significant differences in longitudinal changes from baseline. To test for significant differences between the two treatment groups, the Student's independent $t$ test and the Bonferroni-Holm correction to adjust for multiplicity of testing were used. All tests were two-tailed, with $p<0.05$ considered statistically significant.

\section{Results}

\section{Clinical Characteristics}

Twelve patients were randomized to LRYGB and 11 to LSG. All surgical procedures were successfully concluded laparoscopically with no conversion to open surgery. Table 1 provides demographics: Both groups had similar preoperative characteristics, including BMI, a clearly disturbed glucose homeostasis, expressed as elevated fasting glucose and insulin concentrations, and a highly pathological HOMA index, indicating insulin resistance. Normal values for the HOMA index were defined from values obtained in a test series of 60 healthy normal weight persons at our institution. All patients underwent a complete evaluation at 1 week, 3 months, and 1 year postsurgery.

\section{Weight Loss and Glycemic Control}

Both procedures achieved a marked reduction in body weight and BMI (Table 2). LRYGB patients lost slightly more weight at 3 and 12 months, but this was not statistically significant. Accordingly, excessive BMI loss at 12 months was $77 \%$ (LRYGB) and 65.6\% (LSG) (both $p<0.001$ vs. preoperative values, no significant difference between the groups; Table 2). Fasting glucose and insulin levels dropped, and insulin resistance (HOMA index) returned to near normal values at 1 year, with no significant differences between the procedures (Figs. 1 and 2).

\section{Meal-Stimulated Satiety Hormone Secretion}

\section{Upper Gastrointestinal Peptides}

Ghrelin Physiological ghrelin levels in the non-obese are characterized by a rise during fasting periods and a rapid postprandial fall. The postprandial ghrelin inhibition was missing in all our patients preoperatively. One week postoperatively, ghrelin levels were lower than preoperatively in both groups ( $p<0.05$ vs. preop values). Lower ghrelin levels could be observed for fasting and meal-stimulated ghrelin levels as well. However, the decrease was more prominent in the LSG group, both at 1 week and 3 months postoperatively 
Table 2 Weight, BMI, and EBMIL (\%) after LRYGB or LSG

\begin{tabular}{|c|c|c|c|c|c|}
\hline Parameter & Treatment & Preoperative & 1 week & 3 months & 1 year \\
\hline \multirow[t]{2}{*}{ Weight (kg) } & LRYGB & $133.3 \pm 30.8$ & $127.5 \pm 29.8 * *$ & $111.1 \pm 24.3^{* *}$ & $87.3 \pm 24.1 * *$ \\
\hline & LSG & $120.2 \pm 19.8$ & $116.9 \pm 18.7^{*}$ & $104.4 \pm 17.7 * *$ & $86.3 \pm 15.6^{* *}$ \\
\hline \multirow[t]{2}{*}{ BMI $\left(\mathrm{kg} / \mathrm{m}^{2}\right)$} & LRYGB & $47.6 \pm 6.8$ & $45.6 \pm 6.9^{* *}$ & $39.8 \pm 5.8^{* *}$ & $31.1 \pm 7.5^{* *}$ \\
\hline & LSG & $44.7 \pm 5.3$ & $43.4 \pm 5.2^{*}$ & $38.8 \pm 4.8^{* *}$ & $32.0 \pm 5.0 * *$ \\
\hline \multirow[t]{2}{*}{ EBMIL (\%) } & LRYGB & 0 & $9.8 \pm 5.7 * *$ & $35.8 \pm 9.7 * *$ & $77.0 \pm 24.7 * *$ \\
\hline & LSG & 0 & $6.2 \pm 5.7 *$ & $30.9 \pm 9.6^{* *}$ & $65.6 \pm 21.2 * *$ \\
\hline
\end{tabular}

Data are presented as means \pm SD. No significant differences between the two groups at the same time points were detected EBMIL excessive BMI loss

${ }^{*} p \leq 0.01 ;{ }^{*} p \leq 0.001=$ significant differences compared to preoperative within each group

(lower AUC and lower Cmax, Fig. 3a, c; Table 3). Despite the initial fall, fasting ghrelin levels even exceeded preoperative values in the LRYGB group after 1 year, but the physiological response with the typical postprandial fall was reestablished. Contrarily, patients with LSG showed permanently attenuated ghrelin levels after 1 year (although slightly higher than at 3 months), interestingly without postprandial decline.

$C C K$ Both groups showed a normal CCK response after stimulation with the standardized test meal before operation. Postoperatively, patients with LSG as well as with LRYGB had elevated CCK concentrations. The1-week values were markedly increased, but the effect was short-lasting (60 min). The postprandial test meal response 1 year postoperatively revealed a numerically smaller increase in the LRYGB group than that in the LSG group. Differences in maximal CCK concentrations were statistically significant $(p<0.012)$ (Fig. 3b, d; Table 3).

\section{Lower Gastrointestinal Peptides}

GLP-1 Both groups had a defective GLP-1 response to test meal intake before the operation. LRYGB patients exhibited an early marked increase in postprandial GLP-1 levels at

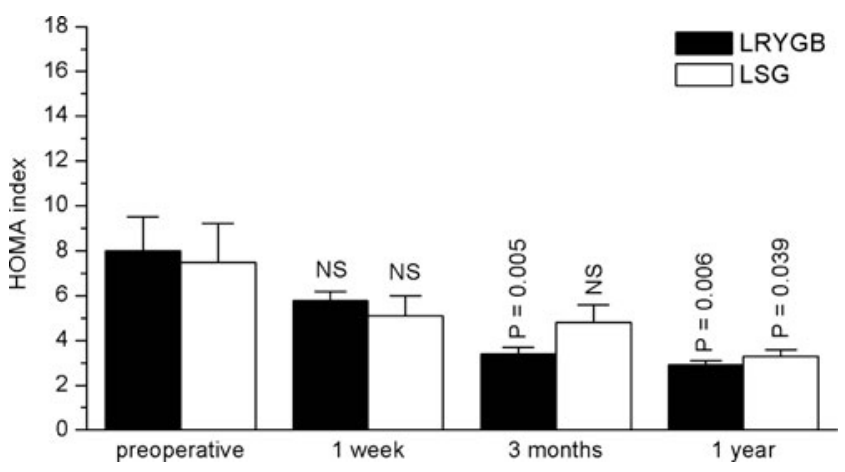

Fig. 1 HOMA index in the two groups of patients (LRYGB and LSG) before, as well as 1 week and 3 and 12 months after the respective operation. Data are means \pm SEM
1 week after this form of bariatric surgery ( $p<0.001$ vs. preop; Fig. 4a, c; Table 3). The markedly increased GLP-1 response was unchanged after 3 months and 1 year in the LRYGB group; a similar but less prominent pattern was seen in the LSG group, although the AUC was numerically clearly smaller in the LSG patients.

Peptide YY Before surgery, PYY levels did not significantly increase in response to food, suggesting a defective PYY response. Fasting PYY levels decreased after surgery in both study groups and expressed an exaggerated postprandial PYY response 1 week after the operation, an effect that was slightly less prominent but still present 3 months and 1 year later (Fig. 4b, d; Table 3). The response pattern and secretory output were comparable, with no significant differences.

\section{Discussion}

Bariatric surgery is the only current treatment option that leads to sustained weight loss and reduction in mortality for morbidly obese patients [1-3]. LRYGB is presently the gold standard, resulting in greater weight loss than purely restrictive procedures $[1,2]$. LSG is a novel bariatric procedure that avoids intestinal bypass. In this prospective, randomized, controlled study, we show that, at 1 year, weight loss and sustained improvement in glycemic control manifest themselves to almost the same extent and at a similar pace after LRYGB and LSG procedures, which are both safe and highly effective therapies for morbid obesity. These outcomes contrast with those following adjustable gastric banding, a purely restrictive procedure [23]. We conclude that LSG might be a reliable alternative to LRYGB and also suggest that foregut exclusion cannot be the sole explanation for the marked weight loss and improvement in glucose metabolism [19-21, 23].

One major focus of this investigation was the time courses of satiety hormones based on their release site (foregut, hindgut). The general consensus is that many of the improvements in 

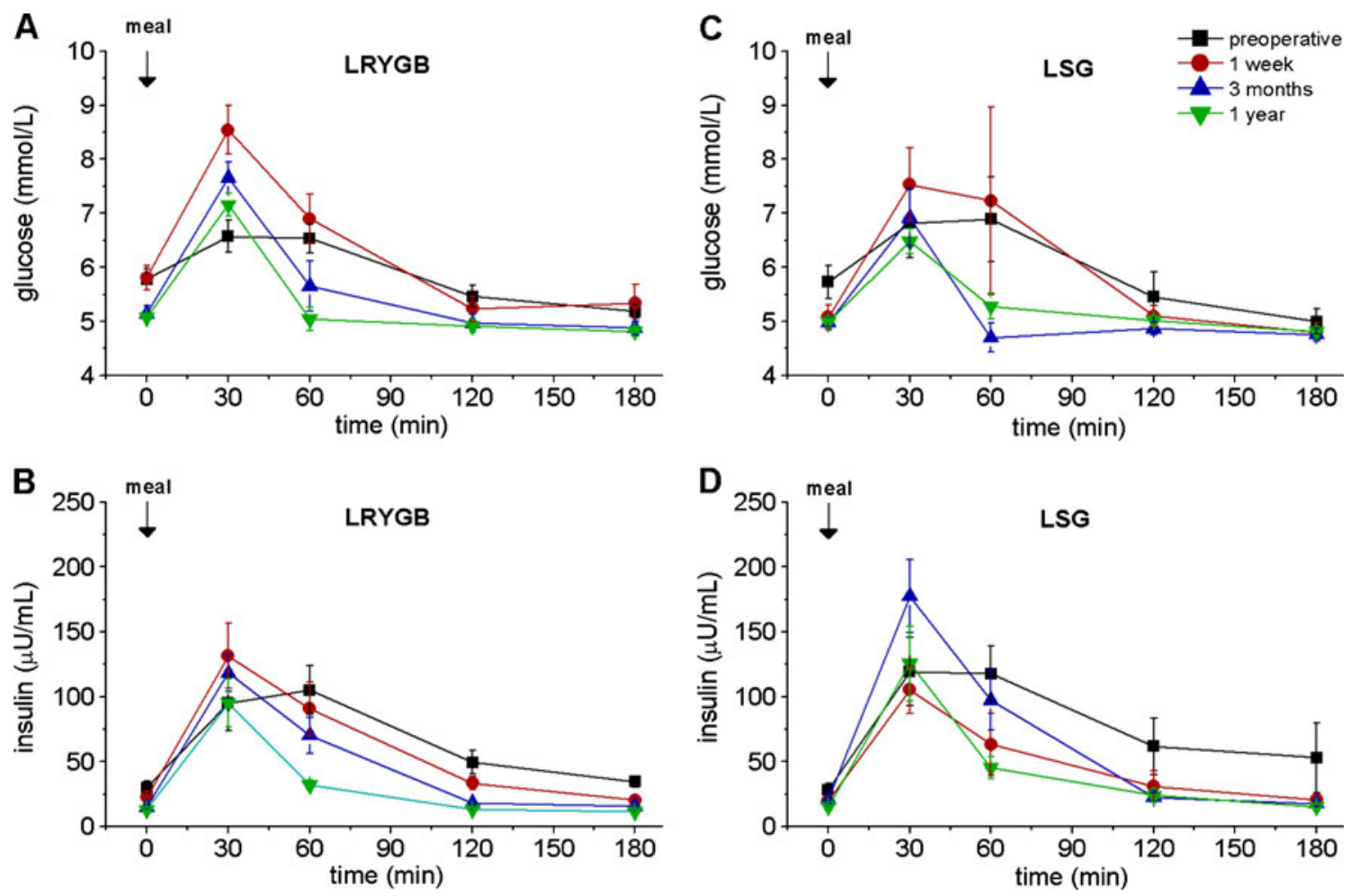

Fig. 2 Fasting and meal-stimulated time courses of glucose and insulin in two groups of patients (LRYGB and LSG) before, as well as 1 week and 3 and 12 months after the respective operation. a Glucose

in the LRYGB group, $\mathbf{b}$ insulin in the LRYGB group, $\mathbf{c}$ glucose in the LSG group, $\mathbf{d}$ insulin in the LSG group. Data are means \pm SEM
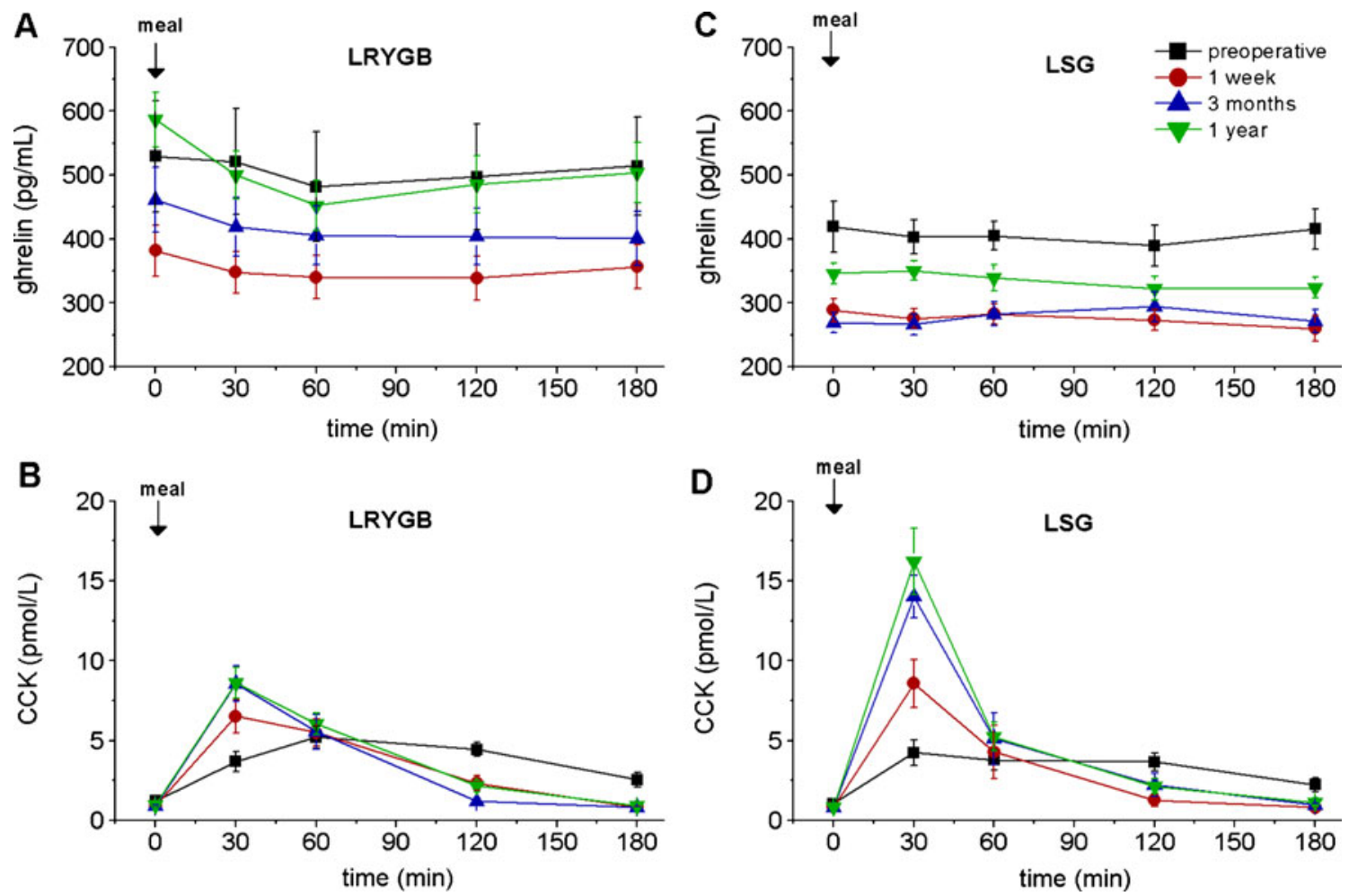

Fig. 3 Fasting and meal-stimulated time courses of ghrelin and CCK in the two groups of patients (LRYGB and LSG) before, as well as 1 week and 3 and 12 months after the respective operation. a Ghrelin in

the LRYGB group, b CCK in the LRYGB group, $\mathbf{c}$ ghrelin in the LSG group, $\mathbf{d}$ CCK in the LSG group. Data are means \pm SEM. Details on the statistical analysis are given in Table 3 
Table 3 Upper (ghrelin and CCK) and lower (GLP-1 and PPY) gastrointestinal peptides after LRYGB or LSG, preoperative and at 1 week, 3 months, and 1 year postsurgery

Data represent means \pm SEM. $p<0.05=$ significant differences between the two groups at the same time points

$N S$ not significant, $A U C$ area under the concentration time profile, Cmax maximum plasma concentrations

$* p \leq 0.05 ; * * p \leq 0.01$;

$* * * p \leq 0.001=$ significant differences compared to the preoperative within group

\begin{tabular}{|c|c|c|c|c|c|}
\hline Parameter & Treatment & Preoperative & 1 week & 3 months & 1 year \\
\hline \multicolumn{6}{|l|}{ Ghrelin } \\
\hline \multirow[t]{3}{*}{$\mathrm{AUC}(\mathrm{ng} \min / \mathrm{mL}$ ) } & LRYGB & $90 \pm 15$ & $62 \pm 6^{*}$ & $74 \pm 8$ & $88 \pm 8$ \\
\hline & LSG & $72 \pm 5$ & $49 \pm 3 * * *$ & $50 \pm 3 * * *$ & $60 \pm 3$ \\
\hline & $p$ value & NS & NS & 0.064 & 0.016 \\
\hline \multirow[t]{3}{*}{$\mathrm{Cmax}(\mathrm{pg} / \mathrm{mL})$} & LRYGB & $567 \pm 86$ & $395 \pm 37 * *$ & $469 \pm 51 *$ & $587 \pm 43$ \\
\hline & LSG & $451 \pm 36$ & $302 \pm 18 * * *$ & $305 \pm 22 * * *$ & $363 \pm 17$ \\
\hline & $p$ value & NS & NS & 0.048 & 0.001 \\
\hline \multicolumn{6}{|l|}{ CCK } \\
\hline \multirow[t]{3}{*}{ 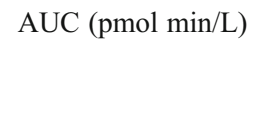 } & LRYGB & $706 \pm 80$ & $622 \pm 84$ & $616 \pm 77$ & $701 \pm 67$ \\
\hline & LSG & $599 \pm 88$ & $562 \pm 122$ & $824 \pm 140 *$ & $897 \pm 105^{* *}$ \\
\hline & $p$ value & NS & NS & NS & NS \\
\hline \multirow[t]{3}{*}{$\mathrm{Cmax}(\mathrm{pmol} / \mathrm{L})$} & LRYGB & $5.6 \pm 0.6$ & $7.2 \pm 1.0$ & $8.7 \pm 1.1 * *$ & $8.7 \pm 0.9^{* *}$ \\
\hline & LSG & $5.0 \pm 0.7$ & $9.3 \pm 1.8$ & $14.3 \pm 1.4 * * *$ & $16.2 \pm 2.1 * * *$ \\
\hline & $p$ value & NS & NS & 0.020 & 0.012 \\
\hline \multicolumn{6}{|l|}{ GLP-1 } \\
\hline \multirow[t]{3}{*}{ AUC $(\mathrm{pmol} \mathrm{min} / \mathrm{L})$} & LRYGB & $326 \pm 54$ & $1,230 \pm 172 * * *$ & $1,381 \pm 183 * * *$ & $1,316 \pm 302 * *$ \\
\hline & LSG & $297 \pm 29$ & $773 \pm 140 * *$ & $998 \pm 160 * *$ & $665 \pm 125^{* *}$ \\
\hline & $p$ value & NS & NS & NS & NS \\
\hline \multirow[t]{3}{*}{$\mathrm{Cmax}(\mathrm{pmol} / \mathrm{L})$} & LRYGB & $3.4 \pm 0.8$ & $15.1 \pm 2.1 * * *$ & $21.2 \pm 3.5^{* * *}$ & $21.2 \pm 5.7^{*}$ \\
\hline & LSG & $2.4 \pm 0.4$ & $10.0 \pm 1.9^{* *}$ & $14.3 \pm 1.9^{* * *}$ & $12.0 \pm 2.2 * *$ \\
\hline & $p$ value & NS & NS & NS & NS \\
\hline \multicolumn{6}{|l|}{ PYY } \\
\hline \multirow[t]{3}{*}{ AUC (ng min/mL) } & LRYGB & $27.6 \pm 2.8$ & $53.5 \pm 2.9 * * *$ & $43.1 \pm 4.1 * *$ & $38.3 \pm 4.3^{*}$ \\
\hline & LSG & $23.6 \pm 1.4$ & $48.7 \pm 5.9 * *$ & $35.2 \pm 3.5^{* *}$ & $31.7 \pm 4.0$ \\
\hline & $p$ value & NS & NS & NS & NS \\
\hline \multirow[t]{3}{*}{ Cmax (pg/mL) } & LRYGB & $175 \pm 18$ & $389 \pm 26^{* * *}$ & $320 \pm 29 * * *$ & $280 \pm 32 * *$ \\
\hline & LSG & $152 \pm 10$ & $353 \pm 52 * *$ & $270 \pm 24 * *$ & $230 \pm 32 * *$ \\
\hline & $p$ value & NS & NS & NS & NS \\
\hline
\end{tabular}

glycemic control achieved by bariatric surgery are likely to be associated with alterations in the secretion of hormones from the gut. LSG restricts the volume capacity of the stomach; in contrast, the LRYGB procedure excludes food from the stomach and the proximal small intestine, thereby exposing the distal gut to altered chyme $[3,18,21]$. It is most likely that both duodenal exclusion (foregut hypothesis) and rapid exposure of distal small intestine to nutrients (hindgut hypothesis) are mechanisms that potentially contribute to improved glycemic control. The two procedures induce slightly different hormonal patterns over time, although the improvement in weight loss, BMI, and glucose homeostasis remains comparable 1 year after surgery. Our observations provide a basis for explaining the procedures' possible mechanisms.

Foregut vs. Hindgut Hypotheses It has been proposed that the anatomical rearrangement alters food passage dynamics, evoking changes in gut hormone secretion to food [3, 19-21, 23]. A variety of studies focusing on hormonal changes after LRYGB have demonstrated decreased ghrelin levels on the one hand and increased levels of GLP-1, PYY336 , and adiponectin after weight loss on the other hand. These changes support the possibility of hormonal weightindependent effects [3, 18-21, 23, 24]. Very few studies have investigated hormonal changes after LSG [14, 15]; here, we document markedly decreased ghrelin levels and increased concentrations of CCK, GLP-1, and PYY3-36.

Consistent with the hindgut hypothesis, RYGB creates a shortcut to the distal small intestine; the stomach is restricted, and chyme is excluded from the foregut. Endogenous GLP-1 and PYY levels were low in both groups before surgery, suggesting diminished endogenous levels of these hormones in obese individuals $[3,18,21]$. Here, postprandial GLP-1 and PYY values dramatically and lastingly increased after RYGB, most likely by direct nutrient contact with distal intestinal L cells. Surprisingly, as this procedure does not alter small intestine food passage, a similar increase in GLP-1 and PYY was seen after LSG. An explanation for this might be that GLP-1 release is not only triggered via direct nutrient contact with distal L cells [25]. Another stimulus for GLP-1 secretion 

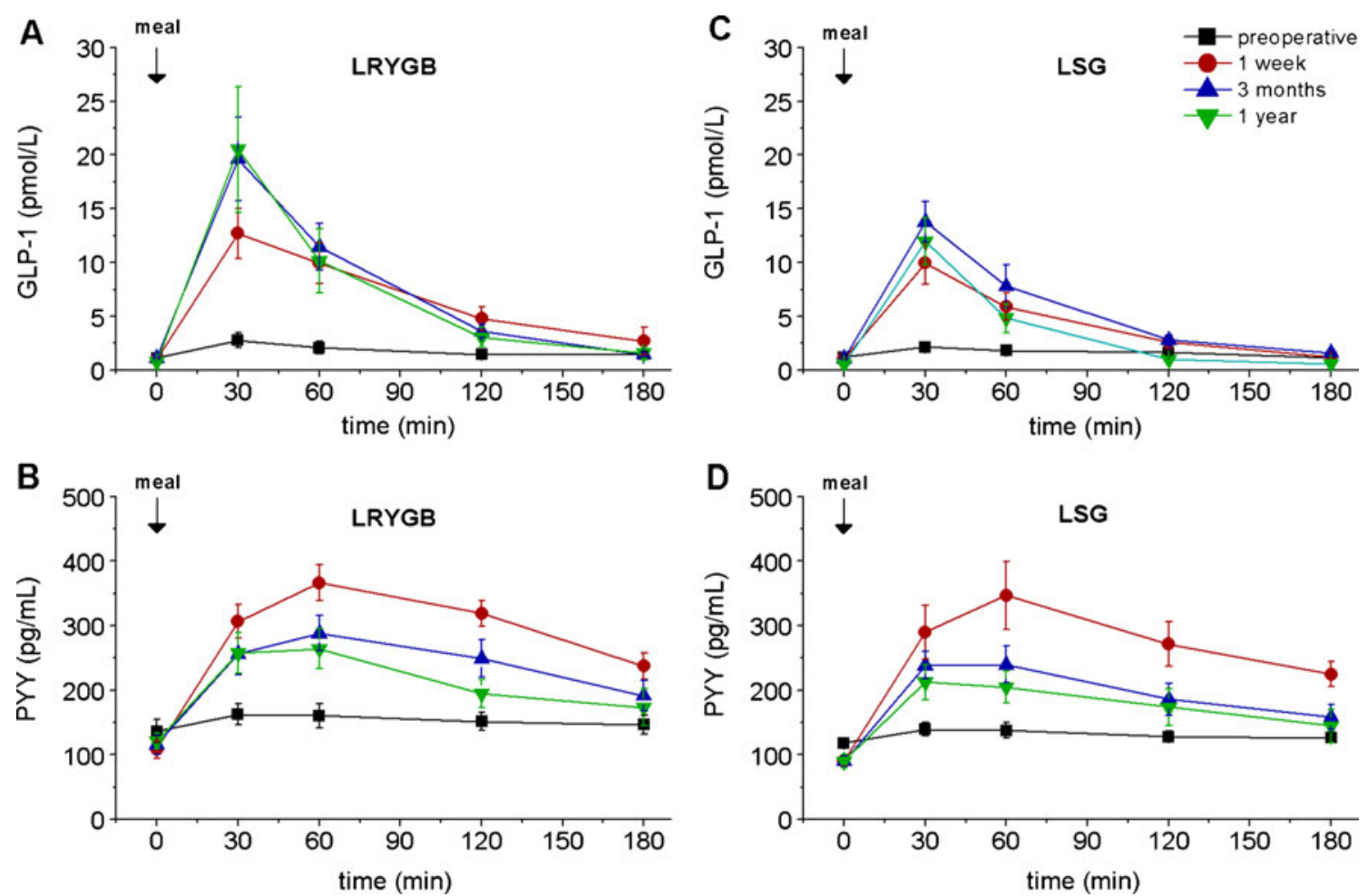

Fig. 4 Fasting and meal-stimulated time courses of GLP-1 and PYY in the two groups of patients (LRYGB and LSG) before, as well as 1 week and 3 and 12 months after the respective operation. a GLP-1 in the

LRYGB group, b PYY in the LRYGB group, $\mathbf{c}$ GLP-1 in the LSG group, $\mathbf{d}$ PYY in the LSG group. Data are means \pm SEM. Details on the statistical analysis are given in Table 3

is derived by proximal nutrient signals, e.g., increased CCK secretion. CCK blood levels are stimulated by long chain free fatty acid formation [26]. This could explain the markedly more pronounced CCK stimulation in the LSG group. As expected, both operations greatly enhanced postprandial GLP-1 and PYY surges. The hormonal changes and weight loss clearly enhanced insulin secretion and sensitivity, as estimated by the HOMA index. Of note, glucose homeostasis began improving 1 week after surgery, even before any meaningful weight loss. Although weight loss is associated with improved glucose control, it has been suggested that postRYGB improvement in glucose metabolism is greater than with equivalent weight loss from other regimens [3, 5-8, 21, 27]. Here, we provide experimental evidence that improvement in glucose control is similar with LSG as well as with LRYGB.

Furthermore, at 1 year follow-up, BMI values were similar with both procedures, and the changes paralleled improvements in dyslipidemia (lower triglycerides, increased HDL cholesterol levels) [28]. The LSG results are interesting: In contrast to adjustable gastric banding, which like LSG is a gastric restrictive procedure, ameliorated glycemic control was observed already at 1 week postoperatively, even before substantial weight loss occurred. These findings concur with results from Lee et al., who compared matching patients with moderate obesity (BMI $27-35 \mathrm{~kg} / \mathrm{m}^{2}$ ) but poorly controlled

diabetes undergoing either sleeve gastrectomy or a sleeved version of RYGB. After 6 months, both procedures achieved equivalent weight loss and improvement in glycemic control $[29,30]$.

A potential explanation for PYY and GLP-1 increases following LSG could be accelerated gastric emptying and earlier contact of chyme with the L cells of the hindgut. Scintigraphic studies performed up to 2 years after LSG showed accelerated gastric emptying for solid and liquid foods [31, 32]. In contrast, a recent MRI study, focused on the motility changes postoperatively, could demonstrate two different functional regions in the remnant stomach: The antral motility remained unchanged even very early postoperatively, whereas the sleeved stomach seemed to be nearly aperistaltic even $6-8$ months postoperatively [33].

The clinical importance of increased PYY levels is unclear: over-expression of PYY in transgenic mice did not change weight or food intake, but other studies have proposed that pharmacologic concentrations of PYY function as anorexic signals, reducing food intake, body weight, and body fat mass $[34,35]$. In summary, glycemic control improved in both groups equally over a 1-year follow-up period, associated with marked increases in GLP-1 (although slightly more so in the RYGB group) and PYY secretions and changes in mealstimulated CCK release. 
Ghrelin Hypothesis The relationship between bariatric surgery and ghrelin levels is controversial. Some years ago, Cummings and co-workers provided initial evidence for reduced secretion of the orexigenic, prodiabetic, foregut hormone, ghrelin, contributing to the anorexic and antidiabetic effects of RYGB [36]. Other authors have failed to confirm these findings [37]. Here, we present postprandial ghrelin profiles over a 1-year period following both procedures. The first interesting observation is the lack of postprandial suppression of ghrelin levels before surgery in both groups, suggesting that the physiological regulation of ghrelin secretion is, at least, partially lost in morbidly obese subjects. In sleeve gastrectomy patients, ghrelin levels were markedly reduced and remained extremely low for several months after the operation, but showed a small (but still markedly reduced) increase in ghrelin after 1 year. This finding is not surprising because the stomach produces the majority of ghrelin. In contrast, ghrelin levels were reduced in post-RYGB patients, but not as pronouncedly so as with LSG. Over time, ghrelin levels returned to preoperative levels but with a major difference: Patients had regained the physiologic postprandial suppression after meal ingestion 1 year after surgery.

The changes in ghrelin can contribute to the marked decrease in weight loss, appetite, and food intake that follows both surgical procedures, but should be more prominent after the LSG procedure [7, 36]. Recent studies in mice deficient for both ghrelin and its receptor indicate that a complete functional absence of ghrelin signaling is sufficient to decrease body weight and fat mass [38-40]. The changes in ghrelin should also contribute to improved glucose homeostasis, as ghrelin can stimulate insulin counter-regulatory hormones, suppress the insulin-sensitizing adipokine, adiponectin, and inhibit insulin secretion [28, 38-40]. From these results, we infer that part of the glycemic improvement after LSG arises from reduced ghrelin secretion.

Our observations are intriguing and to a certain extent unexpected as neither the upper (foregut) nor the distal (hindgut) intestinal hypothesis can fully account for improvement in glucose homeostasis. These results suggest rather that the balance between foregut (ghrelin, CCK) and hindgut hormones (GLP-1, PYY) is a key for understanding the improved glucose homeostasis. Another important factor for the improvement of glucose homeostasis is nutrient sensing and metabolism influencing insulin sensitivity, thus supporting the nutrient-related hormone release and balance. To completely understand the effects and interactions how different bariatric procedures influence nutrient-sensing regulatory mechanisms, influence gut hormone balance, and finally affect glucose homeostasis, more research is warranted.
Acknowledgments We thank Kathleen Bucher and Phillip Hendrickson for editorial assistance. We are indebted to Luisa Baselgia and the team at the Clinical Research Center for excellent assistance in performing the meal studies and Gerdien Gamboni for expert technical assistance in the laboratory. This research was supported by grants from the Swiss National Science Foundation (grant nos. 320000-118330 and 320000-120020), a grant from the Stiftung zur Förderung der Forschung in der Gastroenterologie, and by a grant from Ethicon Endosurgery GmbH, Europe.

Disclosures RP has received unrestricted grants from and consults for Ethicon Endosurgery GmbH, Europe. CB has received an unrestricted grant from Hoffmann-LaRoche, Basel, Switzerland. All other authors declare that they have no conflict of interest.

Open Access This article is distributed under the terms of the Creative Commons Attribution License which permits any use, distribution, and reproduction in any medium, provided the original author(s) and the source are credited.

\section{References}

1. Sjostrom L, Lindroos AK, Peltonen M, et al. Lifestyle, diabetes, and cardiovascular risk factors 10 years after bariatric surgery. N Engl J Med. 2004;351:2683-93.

2. Buchwald H, Avidor Y, Braunwald E, et al. Bariatric surgery: a systematic review and meta-analysis. JAMA. 2004;292:1724-37.

3. Cummings DE, Overduin J, Foster-Schubert KE, et al. Role of the bypassed proximal intestine in the anti-diabetic effects of bariatric surgery. Surg Obes Relat Dis. 2007;3:109-15.

4. MacDonald Jr KG, Long SD, Swanson MS, et al. The gastric bypass operation reduces the progression and mortality of non-insulindependent diabetes mellitus. J Gastrointest Surg. 1997;1:213-20. discussion 220.

5. Pories WJ, Swanson MS, MacDonald KG, et al. Who would have thought it? An operation proves to be the most effective therapy for adult-onset diabetes mellitus. Ann Surg. 1995;222:339-50. discussion 350-332.

6. Schauer PR, Burguera B, Ikramuddin S, et al. Effect of laparoscopic Roux-en Y gastric bypass on type 2 diabetes mellitus. Ann Surg. 2003;238:467-84. discussion 484-465.

7. Thaler JP, Cummings DE. Minireview: hormonal and metabolic mechanisms of diabetes remission after gastrointestinal surgery. Endocrinology. 2009;150:2518-25.

8. Kim S, Richards WO. Long-term follow-up of the metabolic profiles in obese patients with type 2 diabetes mellitus after Roux-en-Y gastric bypass. Ann Surg. 2010;251:1049-55.

9. Adams TD, Gress RE, Smith SC, et al. Long-term mortality after gastric bypass surgery. N Engl J Med. 2007;357:753-61.

10. Nguyen NT, Root J, Zainabadi K, et al. Accelerated growth of bariatric surgery with the introduction of minimally invasive surgery. Arch Surg. 2005;140:1198-202. discussion 1203.

11. Shi X, Karmali S, Sharma AM, et al. A review of laparoscopic sleeve gastrectomy for morbid obesity. Obes Surg. 2010;20:1171-7.

12. Gagner M, Gumbs AA, Milone L, et al. Laparoscopic sleeve gastrectomy for the super-super-obese (body mass index $>60 \mathrm{~kg} / \mathrm{m}(2)$ ). Surg Today. 2008;38:399-403.

13. Nocca D, Krawczykowsky D, Bomans B, et al. A prospective multicenter study of 163 sleeve gastrectomies: results at 1 and 2 years. Obes Surg. 2008;18:560-5.

14. Karamanakos SN, Vagenas K, Kalfarentzos F, et al. Weight loss, appetite suppression, and changes in fasting and postprandial 
ghrelin and peptide-YY levels after Roux-en-Y gastric bypass and sleeve gastrectomy: a prospective, double blind study. Ann Surg. 2008;247:401-7.

15. Peterli R, Wolnerhanssen $B$, Peters $T$, et al. Improvement in glucose metabolism after bariatric surgery: comparison of laparoscopic Roux-en-Y gastric bypass and laparoscopic sleeve gastrectomy: a prospective randomized trial. Ann Surg. 2009;250:234-41.

16. Menenakos E, Stamou KM, Albanopoulos K, et al. Laparoscopic sleeve gastrectomy performed with intent to treat morbid obesity: a prospective single-center study of 261 patients with a median follow-up of 1 year. Obes Surg. 2010;20:276-82.

17. Shah S, Shah P, Todkar J, et al. Prospective controlled study of effect of laparoscopic sleeve gastrectomy on small bowel transit time and gastric emptying half-time in morbidly obese patients with type 2 diabetes mellitus. Surg Obes Relat Dis. 2010;6:152-7.

18. Cummings DE, Overduin J, Foster-Schubert KE. Gastric bypass for obesity: mechanisms of weight loss and diabetes resolution. $\mathbf{J}$ Clin Endocrinol Metab. 2004;89:2608-15.

19. Rubino F, Forgione A, Cummings DE, et al. The mechanism of diabetes control after gastrointestinal bypass surgery reveals a role of the proximal small intestine in the pathophysiology of type 2 diabetes. Ann Surg. 2006;244:741-9.

20. Rubino F, Gagner M, Gentileschi P, et al. The early effect of the Roux-en-Y gastric bypass on hormones involved in body weight regulation and glucose metabolism. Ann Surg. 2004;240:23642.

21. le Roux CW, Aylwin SJ, Batterham RL, et al. Gut hormone profiles following bariatric surgery favor an anorectic state, facilitate weight loss, and improve metabolic parameters. Ann Surg. 2006;243:10814.

22. Gass M, Beglinger C, Peterli R. Metabolic surgery-principles and current concepts. Langenbecks Arch Surg. 2011;396:949-72.

23. Rubino F, Schauer PR, Kaplan LM, et al. Metabolic surgery to treat type 2 diabetes: clinical outcomes and mechanisms of action. Annu Rev Med. 2010;61:393-411.

24. Garcia de la Torre N, Rubio MA, Bordiu E, et al. Effects of weight loss after bariatric surgery for morbid obesity on vascular endothelial growth factor-A, adipocytokines, and insulin. J Clin Endocrinol Metab. 2008;93:4276-81.

25. Gaylinn BD, Thorner MO. Luminal influences to orchestrate gastroenterological hormone secretion: the fat, long-chain fatty acid, cholecystokinin, glucagon-like peptide 1 axis. J Clin Endocrinol Metab. 2010;95:503-4.
26. Beglinger S, Drewe J, Schirra J, et al. Role of fat hydrolysis in regulating glucagon-like peptide-1 secretion. J Clin Endocrinol Metab. 2010;95:879-86.

27. Sugerman HJ, Wolfe LG, Sica DA, et al. Diabetes and hypertension in severe obesity and effects of gastric bypass-induced weight loss. Ann Surg. 2003;237:751-6.

28. Woelnerhanssen B, Peterli R, Steinert RE et al. Effects of postbariatric surgery weight loss on adipokines and metabolic parameters: comparison of laparoscopic Roux-en-Y gastric bypass and laparoscopic sleeve gastrectomy - a prospective randomized trial. Surg Obes Relat Dis 2011.

29. Lee WJ, Chong K, Lee YC, et al. Effects of obesity surgery on type 2 diabetes mellitus Asian patients. World J Surg. 2009;33:1895-903.

30. Lee WJ, Ser KH, Chong K, et al. Laparoscopic sleeve gastrectomy for diabetes treatment in nonmorbidly obese patients: efficacy and change of insulin secretion. Surgery. 2010;147:664-9.

31. Melissas J, Daskalakis M, Koukouraki S, et al. Sleeve gastrectomy —a "food limiting" operation. Obes Surg. 2008;18:1251-6.

32. Melissas J, Koukouraki S, Askoxylakis J, et al. Sleeve gastrectomy: a restrictive procedure? Obes Surg. 2007;17:57-62.

33. Baumann T, Kuesters S, Grueneberger J, et al. Time-resolved MRI after ingestion of liquids reveals motility changes after laparoscopic sleeve gastrectomy — preliminary results. Obes Surg. 2011;21:95-101.

34. Batterham RL, Cowley MA, Small CJ, et al. Gut hormone PYY(336) physiologically inhibits food intake. Nature. 2002;418:650-4.

35. Boey D, Lin S, Enriquez RF, et al. PYY transgenic mice are protected against diet-induced and genetic obesity. Neuropeptides. 2008;42:19-30.

36. Cummings DE, Weigle DS, Frayo RS, et al. Plasma ghrelin levels after diet-induced weight loss or gastric bypass surgery. N Engl J Med. 2002;346:1623-30.

37. Garcia-Fuentes E, Garrido-Sanchez L, Garcia-Almeida JM, et al. Different effect of laparoscopic Roux-en-Y gastric bypass and open biliopancreatic diversion of Scopinaro on serum PYY and ghrelin levels. Obes Surg. 2008;18:1424-9.

38. Wortley KE, del Rincon JP, Murray JD, et al. Absence of ghrelin protects against early-onset obesity. J Clin Invest. 2005;115:3573-8.

39. Zigman JM, Nakano Y, Coppari R, et al. Mice lacking ghrelin receptors resist the development of diet-induced obesity. J Clin Invest. 2005; 115:3564-72.

40. Kirchner H, Tong J, Tschop MH, et al. Ghrelin and PYY in the regulation of energy balance and metabolism: lessons from mouse mutants. Am J Physiol Endocrinol Metab. 2010;298:E909-19. 